\title{
Removal of scattered light in the Earth atmosphere
}

\author{
Sonoyo Mukai ${ }^{1}$, Itaru Sano ${ }^{1}$, and Anthony Toigo ${ }^{2}$ \\ ${ }^{1}$ Faculty of Science and Technology, Kinki University, 3-4-1 Kowakae, Higashi-Osaka 577-8502, Japan \\ ${ }^{2}$ California Institute of Technology, U.S.A.
}

(Received October 6, 1997; Revised February 26, 1998; Accepted March 6, 1998)

\begin{abstract}
Atmospheric correction algorithm, which means a procedure to remove scattered light in an atmosphere from the spaced-based data, are shown for ocean color data given by the satellite ADEOS. In order to achieve better atmospheric correction, this paper proposes two subjects; one is how to determine aerosol characteristics by referring to both of radiance and polarization, and the other is introduction of atmospheric correction coefficients.

At first it is shown that a heterogeneous grain model according to Maxwell-Garnett mixing rule as small watersoluble (WS) inclusions in an oceanic (OC) matrix is available to interpret ADEOS/OCTS and POLDER data observed over the Arabian Sea. Our algorithm is based on an idea that aerosol characteristics can be estimated in terms of scattering behavior in the polarization field. Then atmospheric correction, which is based on radiative transfer process in an atmosphere-ocean model involving the retrieved aerosol model, is applied to ocean color data given by ADEOS/OCTS. Finally our atmospheric correction provides an expected chlorophyll map near the sea surface.

It is of interest to mention that retrieval of atmospheric aerosols is improved by combination use of radiance and polarization, moreover atmospheric correction process is progressed by using the correction coefficients.
\end{abstract}

\section{Introduction}

Ocean color data given by the Ocean Color and Temperature Scanner (OCTS) onboard the Advance Earth Observing Satellite (ADEOS) is considered here. ADEOS was unfortunately ten-months life but provided us with valuable information of Earth environments since August 17 in 1996(NASDA, 1996). The OCTS is the second ocean color sensor in eighteen years since the first Nimbus-7/CZCS (Coastal Zone Color Scanner) (Zion, 1983). Furthermore ocean color remote sensing is expected to progress with SeaWiFS of the USA, which was successfully launched on August 1 in 1997 (see Gregg et al., 1997). At this time we focus our attention on atmospheric correction for ocean color data.

Atmospheric correction is the process of removal of contaminated atmospheric light from space-borne data. This process is a key factor especially for ocean color analysis, because the contribution of atmospheric light to the satellite data is about 80 to $90 \%$ in the visible wavelengths over the ocean. Certainly atmospheric correction is necessary not only for ocean color analysis in the visible wavelengths but also for the longer wavelengths and for land usage (Zagolski and Gastellu-etchegorry, 1995; François and Ottlé, 1994). Since atmospheric light is mainly due to multiple scattering by aerosols, the aerosol model itself contributes greatly to the atmospheric correction of the ocean color data (Mukai et al., 1992; Fukushima and Toratani, 1997). It is well known that atmospheric aerosols reflect the environmental change. Namely aerosol characteristics might change greatly in temporal and regional scales (Dalu et al., 1995; Pilinis et al.,

Copy right(c) The Society of Geomagnetism and Earth, Planetary and Space Sciences (SGEPSS); The Seismological Society of Japan; The Volcanological Society of Japan; The Geodetic Society of Japan; The Japanese Society for Planetary Sciences.
1995; Liu et al., 1996; Carmichael et al., 1997). Therefore the efficiency of atmospheric correction strongly depends on how we can set up an appropriate aerosol model for the satellite-image concerned.

This work partly cites aerosol retrieval by using both of radiance and polarization in the near-infrared wavelengths given by both of ADEOS/OCTS and POLDER. The POLDER (POLarization and Directionality of Earth Reflectance) is one of the sensors on board the satellite ADEOS, and aims to collect global-scale visible and near-infrared observations of polarized and directional solar radiance reflected by the Earth-atmosphere-surface system (Deschamps et al., 1994). It is shown that aerosol retrieval can be efficiently pursued by combination use of radiance with polarization, because the polarization features strongly depend on the optical properties of particles shown by Mukai et al. (1996).

Finally our atmospheric correction is applied for the ADEOS/OCTS ocean color data. Here the radiative transfer process in an atmosphere-ocean model involving the retrieved aerosol model is simulated. We have introduced an idea of atmospheric correction coefficients, which represent the values of a ratio of the water leaving radiance to the observed radiance at the satellite (see Mukai et al., 1992). Namely the atmospherically corrected images are obtained by multiplication of the calculated coefficients with the raw ocean color data. An expected chlorophyll map near the sea surface is derived through bio-optical algorithms.

\section{Aerosol Retrieval Based on ADEOS/OCTS}

It is well known that aerosols contribute greatly in the atmosphere by scattering and absorption in the visible and near infrared region of the spectrum. A procedure to estimate aerosol models suitable to the OCTS image of interest 
is shown first. It is found that, in the near-infrared wavelength of OCTS band $6(0.67 \mu \mathrm{m})$ and band $8(0.865 \mu \mathrm{m})$, the contribution of radiation out of the ocean to total radiation is negligibly small and the optical thickness of the atmosphere is also small, by Sano et al. (1997). In these two bands the contribution of gas absorption is small in comparison with that in the band $7(0.76 \mu \mathrm{m})$, where $\mathrm{O}_{2}$ A-band absorption is too dominant to neglect. Therefore it is possible to assume that the OCTS band 6 and 8 images approximately represent the single scattering pattern by atmospheric constituents. Therefore the OCTS radiance data at bands 6 and 8 are used to retrieve the aerosol characteristics, e.g., size and refractive index. A single scattering phase matrix is expressed by Stokes parameters $(I, Q, U, V)$ and composed of Rayleigh scattering by molecular gases and Mie scattering by aerosols. The values of the phase matrix elements strongly depend on the characteristics of aerosols because of the uniform distribution of molecular gases. A singlemode log-normal representation with two parameters, the mode radius $(\bar{r})$ and the width of the log-normal curve $(\sigma)$,
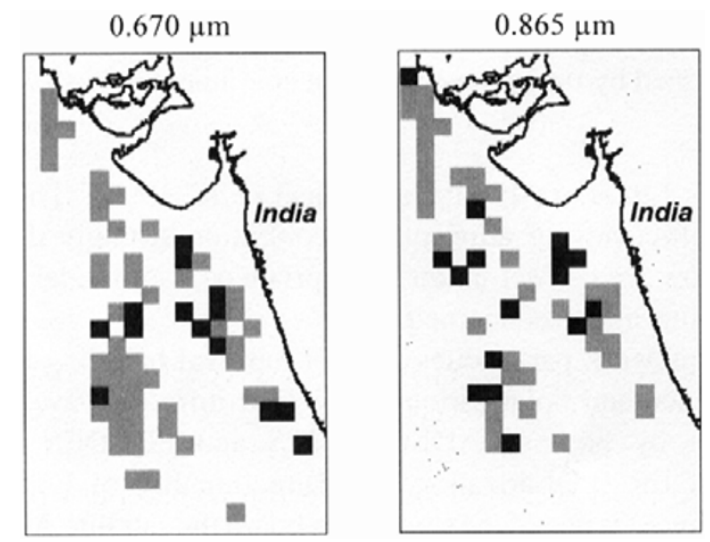

Fig. 1. Correlation coefficients between OCTS data observed in the Arabian Sea on November 18 in 1996 and simulations by using a heterogeneous aerosol model-B at wavelengths of $0.67 \mu \mathrm{m}$ and $0.865 \mu \mathrm{m}$.

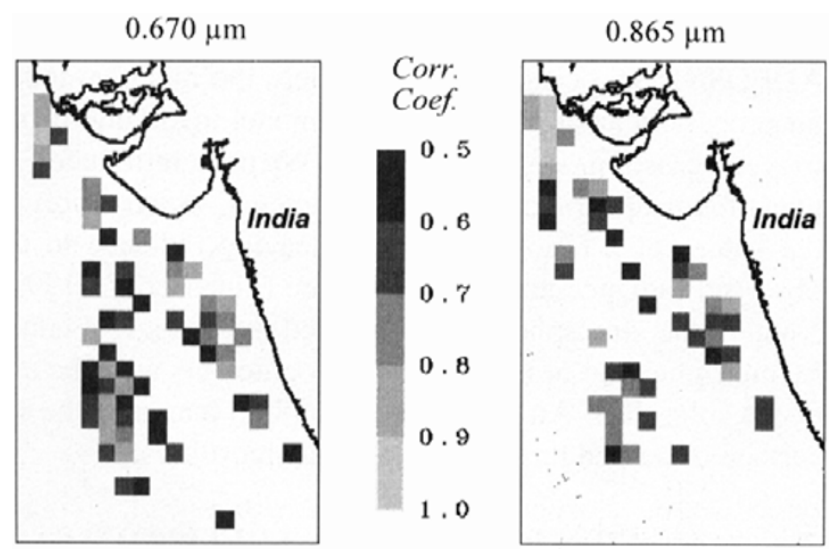

Fig. 2. The best aerosol model of several samples for each window in Fig. 1 at wavelengths of $0.67 \mu \mathrm{m}$ and $0.865 \mu \mathrm{m}$, where black and gray represent the model-A and model-B, respectively. is considered here for one of the most widely used size distributions. Thus it becomes our purpose to retrieve $(\bar{r}, \sigma)$ for the log-normal distribution.

It is natural to consider that several kinds of aerosols could exist together in general. In this section, we take into account heterogeneous particles for maritime aerosol models. World Meteorological Organization (WCP-112, 1986) provides a standard maritime aerosol model, which is mainly composed of oceanic (OC) and water-soluble (WS) components. Oceanic type denotes sea-salt solution in water, and water-soluble type includes water-soluble substances consisting of ammonium sulfate, calcium sulfate and organic compounds. That is, we assume now the maritime aerosols are composed of oceanic component and water-soluble one. The mixing rules of several components into a spherical shaped heterogeneous particle have been presented by several authors, e.g. Maxwell-Garnett (MG) theory (Bohren and Wickramasinghe, 1977), Bruggeman theory (Chýlek and

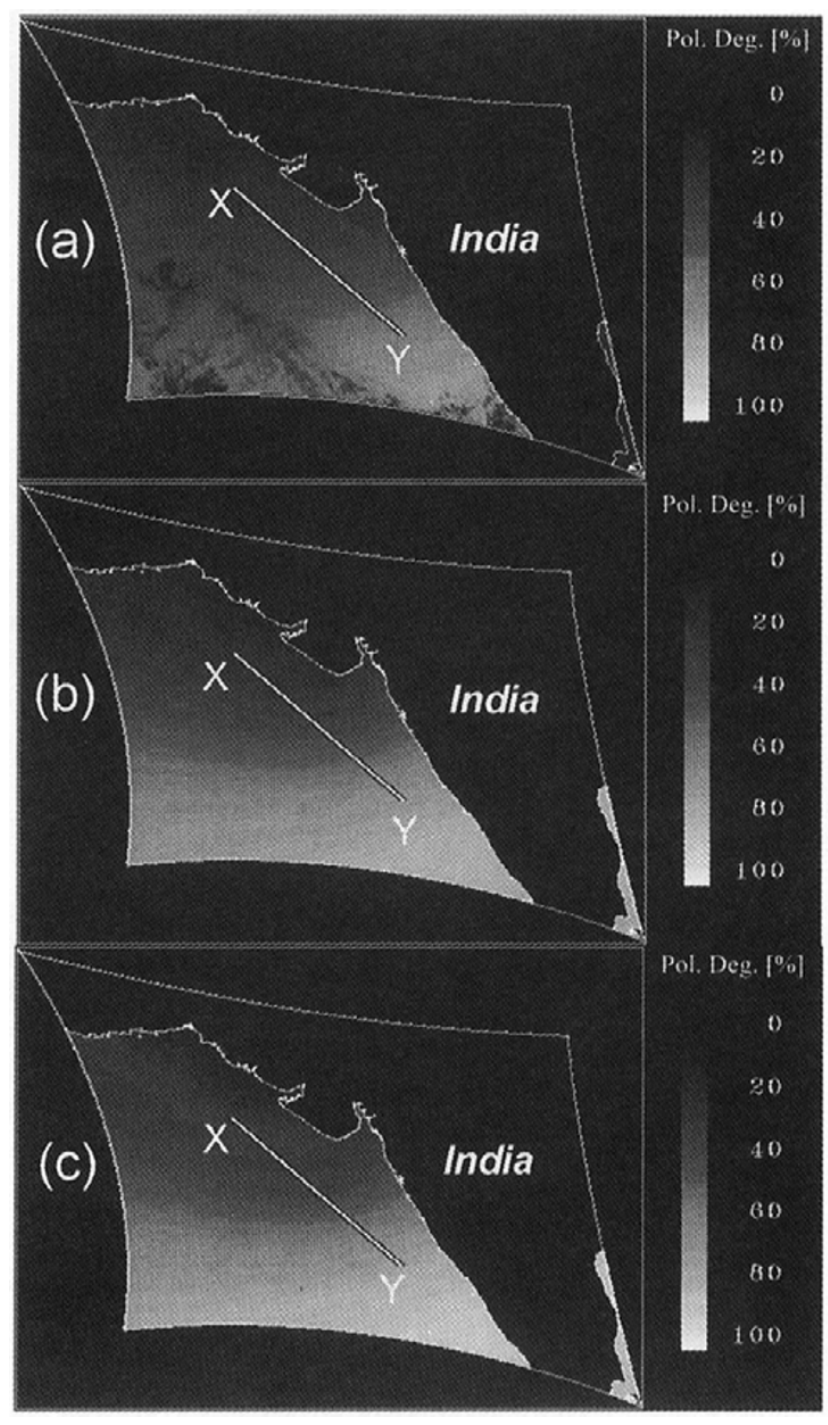

Fig. 3. Distribution of polarization degree at a wavelength of $0.67 \mu \mathrm{m}$. (a) POLDER data observed on Nov. 18, 1996 in the Arabian Sea, (b) Simulated results by using an aerosol model-A, (c) Simulated results by using an aerosol model-B. 


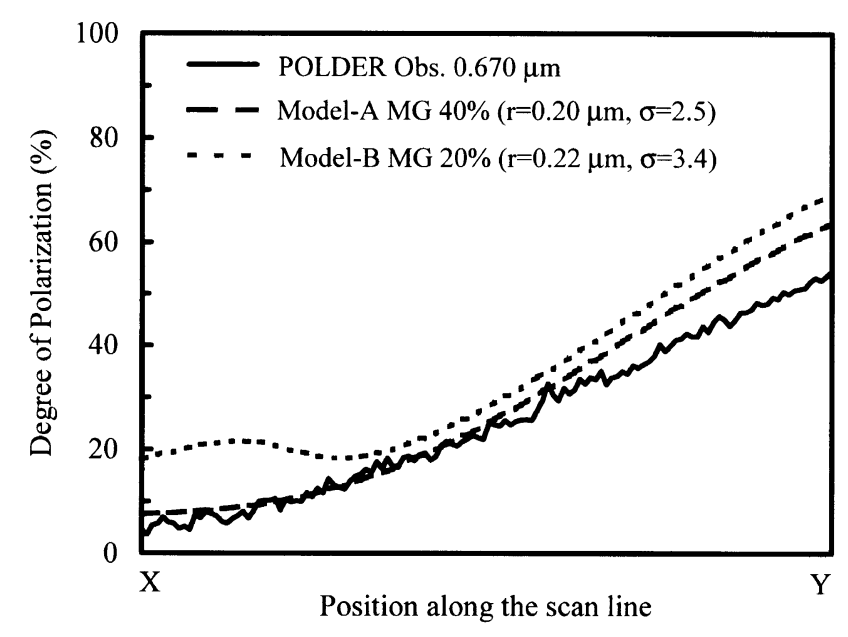

Fig. 4. Polarization degree along the scan line X-Y in Fig. 3, where the solid, dashed and dotted curves represent the values in Fig. 3 (a), (b) and (c), respectively.

Srivastava, 1983; Burkhard, 1984) and core-mantle type (Van de Hulst, 1957). Since these mixing rules have been already adopted for astrophysical grains, descriptions of each theory are omitted and MG theory is employed in our present simulations. That is, a heterogeneous grain model according to Maxwell-Garnett mixing rule as small water-soluble (WS) inclusions in an oceanic (OC) matrix where the volume fraction of WS-inclusions is represented by $f$, is examined for atmospheric aerosols.

This implies that the correlation coefficients between the radiance of OCTS-band 6 and 8 data and the scattering simulations by atmospheric constituents, i.e. Rayleigh scattering by molecules and the heterogeneous model aerosols, become a good indicator to select the appropriate aerosol model for the OCTS images. The detailed descriptions of this algorithm have been shown in our previous paper (Mukai et al., 1992), however brief outline is surveyed again. First we calculate the correlation coefficients between satellite radiance data and the simulated results in respect of directional information. Namely different viewing angles of Sun and satellite on each pixel of the image correspond to light from different angles of incidence to different angles of scattering as far as the light scattering process at the particle is concerned. In other words, each pixel of the image gives each scattering angle. On the other hand, the scattering pattern can be calculated for an arbitrary scattering angle. Thus a scattering angle correlates radiance of a pixel of OCTS bands 6 and 8 images with a numerical value of the scattering pattern of an aerosol type. Note that Rayleigh scattering by molecular gases is also taken into account. The optical thickness of the Rayleigh scattering constituents and absorbent constituents such as ozone and water vapor are obtained by LOWTRAN 7 (Kneizys et al., 1988).

The single scattering phase function in our atmosphere model is expressed with the following form;

$$
P(\Theta)=\left(1-f_{\mathrm{g}}\right) P_{\mathrm{a}}(\Theta)+f_{\mathrm{g}} P_{\mathrm{g}}(\Theta),
$$

where $\Theta$ is the scattering angle and subscripts a and $g$ denote aerosols and molecular gases respectively. The functions $P_{\mathrm{a}}$ and $P_{\mathrm{g}}$, respectively, are given by Mie scattering phase function if an aerosol type is fixed, and by the Rayleigh phase function. The value of $f_{\mathrm{g}}$ represents the ratio of the opacity source by molecules to the total one.

Figure 1 presents an example of correlation coefficients calculated for the OCTS image at band 6 and 8 observed in the west coast of India (Arabian Sea) on November 18 in 1996 by using a MG-aerosol model of $\{f=0.2,(\bar{r}=0.22 \mu \mathrm{m}$, $\sigma=3.4)\}$, which is named model-B hereafter. Note that a window size is assumed to be $75 \times 75$, namely a 75-pixels square is adopted for sampling area to calculate correlation coefficients. The values of correlation coefficients higher than 0.5 are divided into five ranges $[0.5,0.6],[0.6,0.7]$, $[0.7,0.8],[0.8,0.9]$ and $[0.9,1.0]$ from black to white.

For each window, it is possible to determine the best set of optical parameters of aerosol, i.e. $\{f,(\bar{r}, \sigma)\}$, by choosing the highest value of correlation coefficients among several sample aerosols. Two of them are selected (see Fig. 2). In this figure gray and Black, respectively, corresponds to model$\mathrm{B}$ and model-A of $\{f=0.4,(\bar{r}=0.20 \mu \mathrm{m}, \sigma=2.51)\}$. At a glance, a correlation degree of each model seems to be similar.

It is well known that the dust particles originated from desert areas usually exist in the west coast of India, but November is an exceptional season to avoid contamination of the desert dust (Herman et al., 1997). So our present results on November 18 also show rather clear sky, that is the retrieved aerosol model is composed of $40 \%$ water-soluble inclusions mixed into oceanic components.

\section{Polarimetric Properties of Aerosols Based on ADEOS/POLDER}

Degree of polarization is a good indicator to derive optical properties of aerosols, because polarization features strongly depend on characteristics of scattering particles (Hansen and Travis, 1974). Thus an efficient algorithm for aerosol retrieval is developed here by considering simulations of the polarization field. Two heterogeneous MG-models selected in the previous section are used as the basis of functions for aerosol models.

Polarization information simultaneously observed by POLDER is used to determine which is better, aerosol modelA or $-\mathrm{B}$, to interpret the OCTS data. Top image in Fig. 3 presents the polarization degree at wavelengths of $0.67 \mu \mathrm{m}$ observed by POLDER on November 18 in 1996 in the Arabian Sea (nearly the same area as OCTS images in Figs. 1 and 2). The middle and bottom images show the simulated results by using model-A and -B. By comparison with these three images, it is found that the middle image is much more similar to the top one. This feature is clearly enhanced in Fig. 4, where the values of polarization degree along the scan line denoted by X-Y in each image in Fig. 3 are shown. The solid, dashed and dotted curves in Fig. 4 represent the values of polarization degree given by observations, and simulations with model-A and model-B, respectively. The dashed curve applying model-A aerosol coincides with the POLDER data denoted by the solid curve, because model-B (the dotted curve) shows an unexpected hump. Thus we can conclude that model-A is a better candidate than model-B for an aerosol 


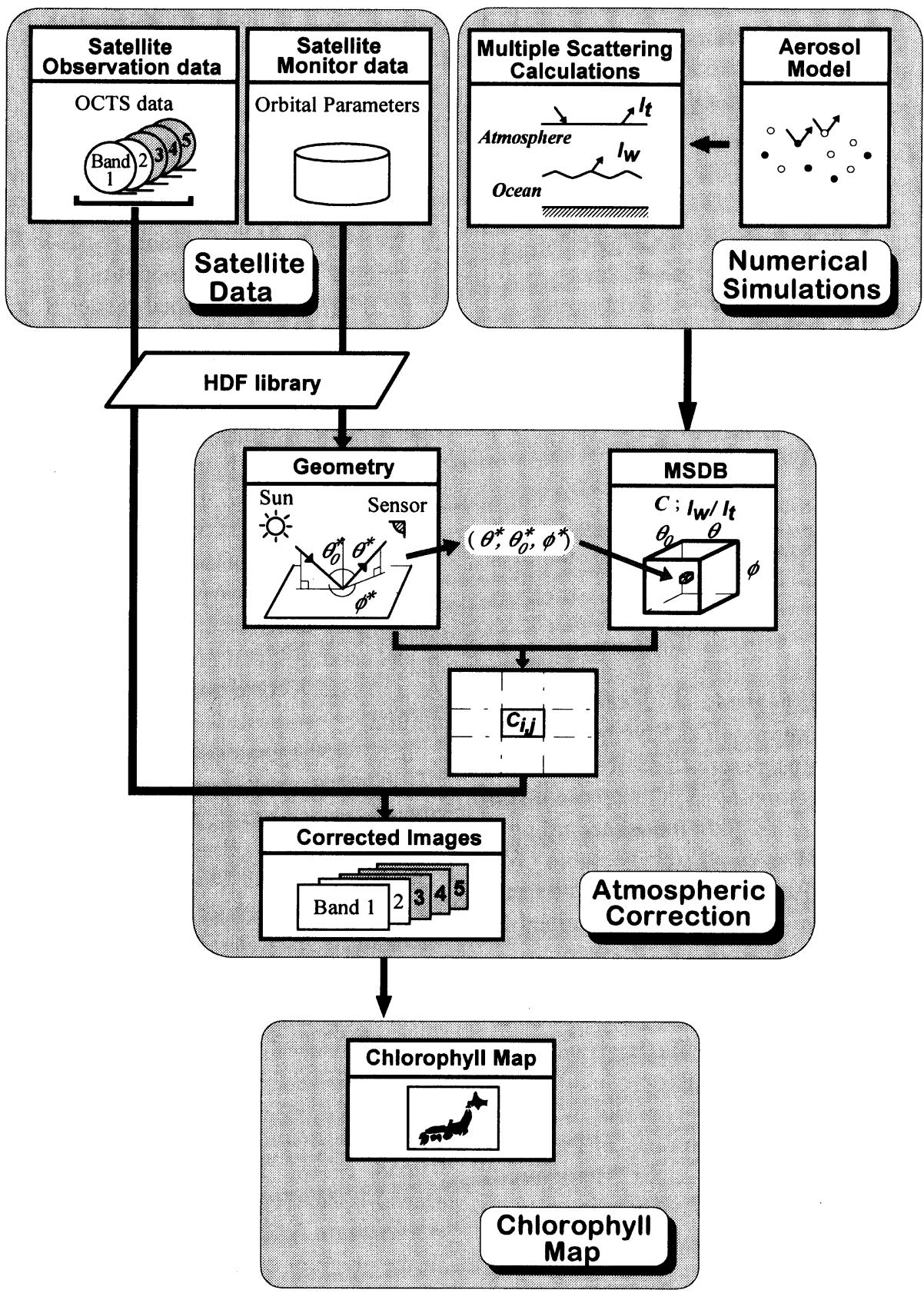

Fig. 5. A system flow of data processing line from OCTS CCT data to the chlorophyll map near the sea surface, where $I$ represnts the specific intensity of radiation in an atmosphere-ocean model and $C$ denotes the atmospheric correction coefficient.

model in order to explain both of OCTS- and POLDER-data observed on November 18 in 1996 in the Arabian Sea.

\section{Atmospheric Correction Coefficients}

A system flow of our data-processing from a OCTS CCT data to a distribution of the pigment concentration, which is briefly called chlorophyll map hereafter, is presented in Fig. 5. As shown in this figure, our atmospheric correction is based on multiple scattering calculations. Therefore the radiative transfer problem in an atmosphere-ocean model involving the retrieved aerosol model has to be numerically solved to obtain the values of the atmospheric correction coefficients $C$. The value of $C$ is obtained from the ratio of the water leaving radiance to the observed radiance at the satellite. Namely $C=I_{\mathrm{w}} / I_{\mathrm{t}}$ (refer to Fig. 5). The values of $C$ depend on the place of each pixel in a satellite image as well as on the wavelength. Atmospherically corrected images are obtained by multiplication of $C$-values to the raw satellite data.

In our present calculations, the MG-model-A is employed for an aerosol model. The sea surface is simulated by multiple facets whose slopes vary according to the isotropic Gaussian distribution with respect to wind speed (Cox and Munk, 1954). Wind speed is assumed to be $5 \mathrm{~m} / \mathrm{sec}$ as a typical value for a clear day. Several ocean models have been prepared. For example, one of them is a completely diffused model, 

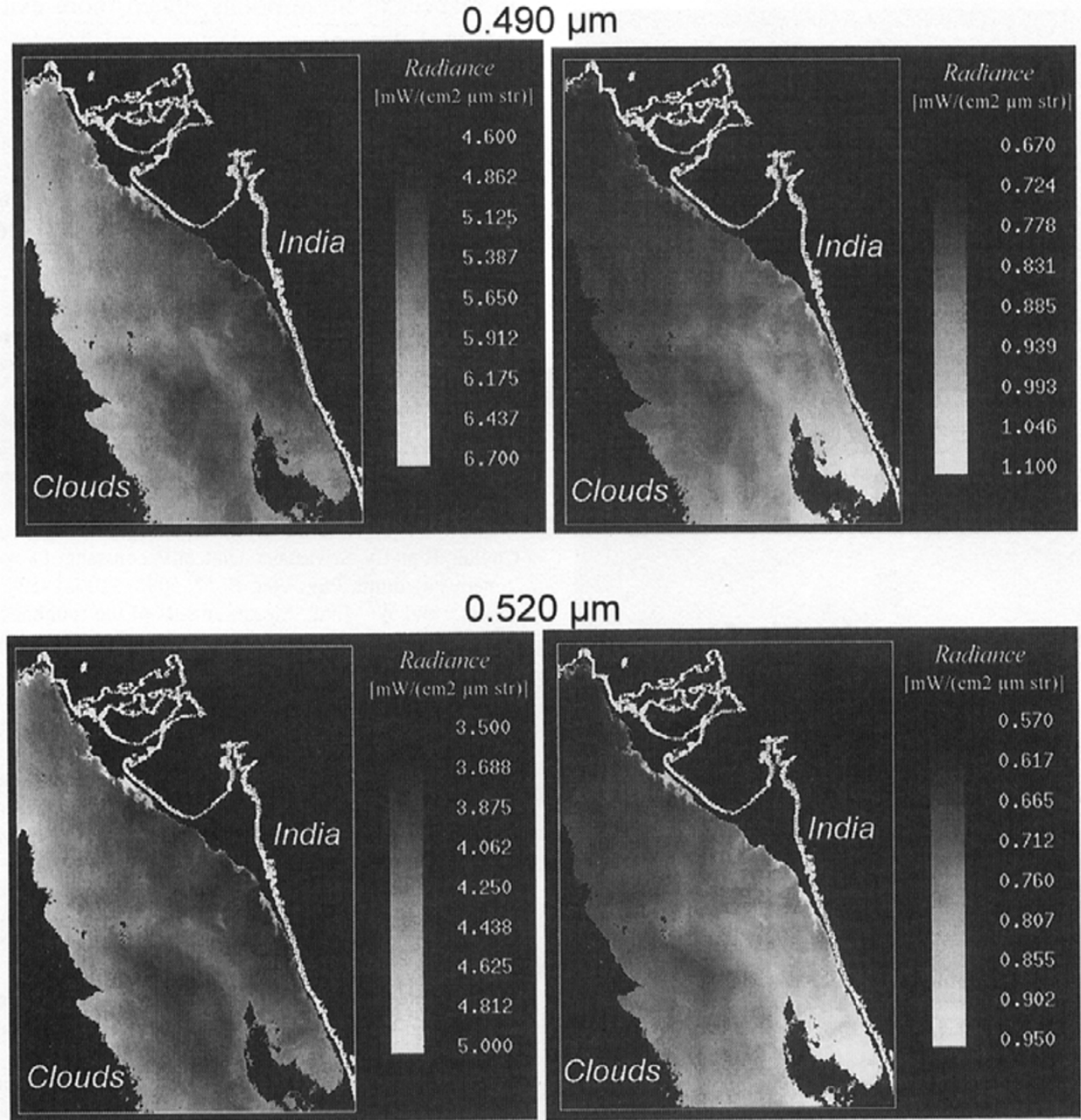

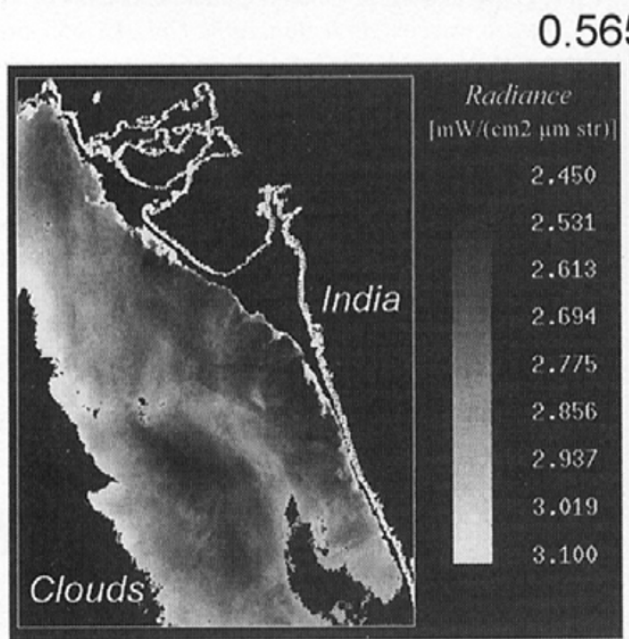

Before

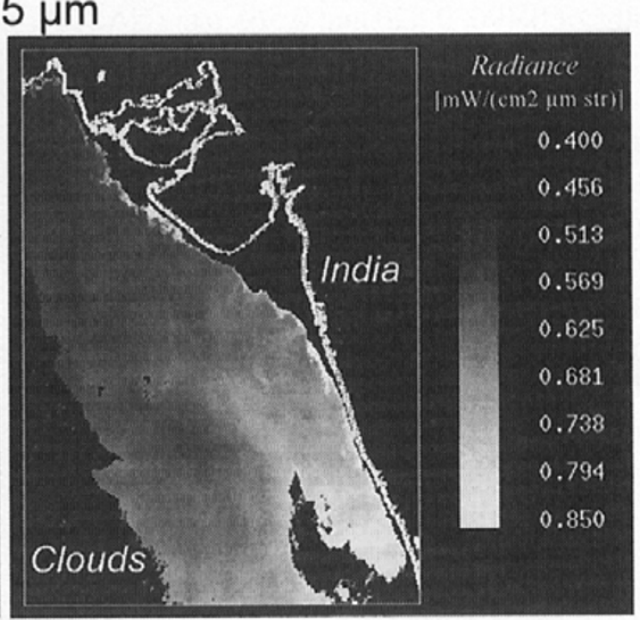

After

Fig. 6. ADEOS/OCTS images of the Arabian Sea on November 18 in 1996 before (left) and after (right) atmospheric correction.

namely upward radiance out of the ocean is represented by Lambert's law. Multiple scattering for the atmosphere-ocean model is solved by the adding-doubling method.

Products through our data processing line in Fig. 5 are shown in Figs. 6 and 7. Expected images after atmospheric correction, which are presented in the right hand side in Fig. 6, are obtained by multiplication of numerical values of $C$ to the raw OCTS images at each wavelength of $0.490,0.520$ and $0.565 \mu \mathrm{m}$, observed on November 18 in 1996 shown in the left side in Fig. 6. We found that the over illumination at 


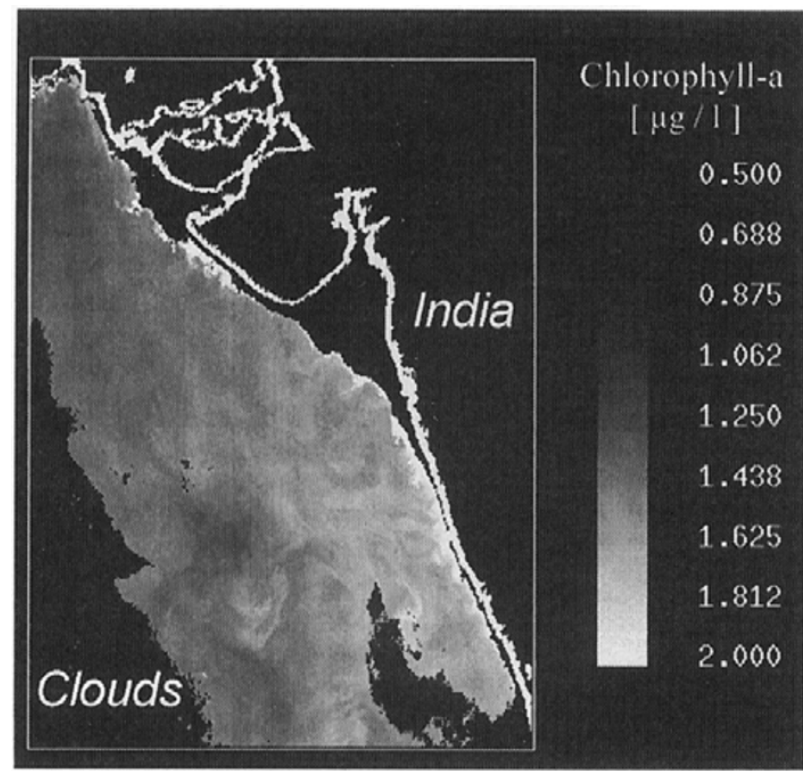

Fig. 7. An expected chlorophyll map by using OCTS data on Nov. 18, 1996 in the Arabian Sea.

the limb of the raw images is due to atmospheric effects and can be eliminated by applying the atmospheric correction algorithm. Figure 7 represents the obtained chlorophyll map in units of $\mu \mathrm{g} / \mathrm{l}$, where bio-optical algorithms are employed by using ocean color data as follows;

$$
\begin{aligned}
& \text { Chlorophyll } \\
& =0.2818\left[\left(\operatorname{Rad}_{0.520}+\operatorname{Rad}_{0.565}\right) / \operatorname{Rad}_{0.490}\right]^{3.497},
\end{aligned}
$$

where Rad represents the radiance after atmospheric correction at wavelengths of $0.490,0.520$ and $0.565 \mu \mathrm{m}$ (NASDA, 1997)

\section{Discussions}

It is found that an efficient atmospheric correction for ocean color data is pursued due to improved aerosol retrieval by using both of radiance and polarization degree and employment of atmospheric correction coefficients.

It is natural to consider that several kinds of aerosol components exist together. But it is difficult to determine the mixing structure of each components. At any rate, from present work, we can say that such a Maxwell-Garnett mixing rule as small water-soluble (WS) inclusions in an oceanic (OC) matrix is available to interpret the space-borne data, and polarization information is useful to improve the aerosol retrieval.

In this paper atmospheric aerosols have been focused, however, we know well that many other subjects should be considered as far as atmospheric correction is concerned, e.g. removal of thin clouds, adjacent effect in the coastal zone, white caps of sea waves, the ocean model itself and so on. We compared the water leaving radiance for several ocean models (Hale and Querry, 1973; Morel, 1974; Tanaka and Nakajima, 1977) and found that the upward intensity at the sea surface level depends upon the ocean model (Masuda and Takashima, 1988). In order to draw the definite conclusions in respect of these points, much more extensive measurements of atmosphere and ocean, and their retrieval algorithms are desired.

Acknowledgments. POLDER data was distributed by CNES in France. This work was partially supported by NASDA (PSPC20170), and also by scientific research funds from the Ministry of Education, Science and Culture of Japan (09780485, 09227223).

\section{References}

Bohren, C. F. and N. C. Wickramasinghe, On the computation of optical properties of heterogeneous grains, Astrophys. Space Sci., 50, 461-472, 1977.

Burkhard, D. G., Frequency dependent dielectric constant and conductivity for a medium containing impurities, Appl. Opt., 23, 2718-2727, 1984.

Carmichael, G. R., M.-S. Hong, H. Ueda, L.-L. Chen, K. Murano, J. K. Park, H. Lee, Y. Kim, C. Kang, and S. Shim, Aerosol composition at Cheju Island, Korea, J. Geophys. Res., 102, 6047-6061, 1997.

Chýlek, P. and V. Srivastava, Dielectric constant of a composite inhomogeneous medium, Phys. Rev. B, 27, 5098-5106, 1983.

Cox, C. and W. Munk, Measurements of the roughness of the sea surface from photographs of the sun's glitter, J. Opt. Soc. Amer., 44, 838-850, 1954

Dalu, G., R. Rao, A. Pompei, G. P. Boi, Tonna, and B. Olivieri, Aerosol optical properties retrieved from solar aureole measurements over southern sardinia, J. Geophys. Res., 100, 26135-26140, 1995.

Deschamps, P. Y., F.-M. Bréon, M. Leroy, A. Podarie, A. Bricaud, J. C. Buriez, and G. Sèze, The POLDER mission: Instrument characteristics and scientific objectives, IEEE Trans. Geosci. Remote Sensing, 32, 598$615,1994$.

François, C. and C. Ottlé, Atmospheric correction in the thermal infrared Global and water vapor dependent split-window algorithms-applications to ATSR and AVHRR data, IEEE Trans. Geosci. Remote Sensing, $\mathbf{3 4}$ 457-470, 1994

Fukushima, H. and M. Toratani, Asian dust aerosol: Optical effect on satellite ocean color signal and a scheme of its correction, J. Geophys. Res. 102, 119-130, 1997.

Gregg, W. W., F. S. Patt, and R. H. Woodward, Development of a simulated data set for the seaWiFS mission, IEEE Trans. Geosci. Remote Sensing, 35, 421-435, 1997.

Hale, G. M. and M. R. Querry, Optical constants of water in the 200-nm to 200- $\mu$ m wavelength region, Appl. Opt., 12, 555-563, 1973.

Hansen, J. H. and L. D. Travis, Light scattering in planetary atmospheres, Space Sci. Rev., 16, 527-610, 1974.

Herman, J. R., P.-K. Bhartia, O. Torres, C. Hsu, C. Seftor, and E. Celarier, Global distribution of UV-absorbing aerosols from Nimbus 7/TOMS data J. Geophys. Res., 102, 16911-16922, 1997.

Kneizys, F. X., E. P. Shettle, L. W. Abreu, J. H. Chetwynd, G. P. Anderson, W. O. Gallery, J. E. A. Selby, and S. A. Clough, Users guide to LOWTRAN 7, Tech. Rep. AFGL-TR-88-0177, Air Force Geophysics Laboratory, Hanscom AFB. MA, 1988.

Liu, P. S. K., W. R. Leaitch, C. M. Banic, S.-M. Li, D. Ngo, and W. J. Megaw, Aerosol observations at Chebogue Point during the 1993 North Atlantic regional Experiment: Relationships among cloud condensation nuclei, size distribution, and chemistry, J. Geophys. Res., 101, 28971-28990, 1996.

Masuda, K. and T. Takashima, Dependence of the radiation just above and below the ocean surface on atmospheric and oceanic parameters, $\mathrm{Appl}$. Opt., 27, 4891-4898, 1988.

Morel, A., Optical properties of pure water and pure sea water, in Optical Aspects of Oceanography, edited by N. G. Jerlov and E. S. Nielsen, 1 pp., Academic Press, London, 1974.

Mukai, S., I. Sano, K. Masuda, and T. Takashima, Atmospheric correction for ocean color remote sensing: Optical properties of aerosols derived from CZCS imagery, IEEE Trans. Geosci. Remote Sensing, 30, 818-824, 1992.

Mukai, S., I. Sano, and T. Takashima, Investigation of atmospheric aerosols based on polarization measurements and scattering simulations, Opt. Rev. 3, 487-491, 1996.

NASDA, ADEOS Reference Hand Book, 338pp., NASDA/EORC, Tokyo, Japan, 1997.

Pilinis, C., S. Pandis, and J. Seinfeld, Sensitivity of direct climate forcing by atmospheric aerosols to aerosol size and composition, J. Geophys. Res., 100, 18739-18754, 1995. 
Sano, I., S. Mukai, and T. Takashima, Polarimetric properties of atmospheric aerosols, Proc. SPIE, 3121, 361-369, 1997.

Tanaka, M. and T. Nakajima, Effects of oceanic turbidity and index of refraction of hydrosols on the flux of solar radiation in the atmosphereocean system, J. Quant. Spectrosc. Radiat. Transfer, 18, 93-111, 1977.

Van de Hulst, H. C., Light Scattering by Small Particles, 470pp., John Wiley, London, 1957.

World Climate Programme, WCP-112, A preliminary cloudless standard atmosphere for radiation computation, WMO/TD-No. 24, World Meteorological Organization, Geneva, 1986.
Zagolski, F. and J. P. Gastellu-etchegorry, Atmospheric correction of AVIRIS images with a procedure based on the inversion of the $5 \mathrm{~S}$ model, Int. J. Remote Sensing, 16, 3115-3146, 1995.

Zion, P. M., Description of algorithms for processing coastal zone color scanner (CZCS) data, NASA/JPL, Pasadena, CA, JPL Pub. 83-98, pp. 129, 1983.

S. Mukai (e-mail: mukai@im.kindai.ac.jp), I. Sano, and A. Toigo 